Delft University of Technology

\title{
Micromechanical validation of a mesomodel for plasticity in composites
}

Van Der Meer, Frans P.

DOI

10.1016/j.euromechsol.2016.06.008

Publication date

2016

Document Version

Accepted author manuscript

Published in

European Journal of Mechanics A - Solids

\section{Citation (APA)}

Van Der Meer, F. P. (2016). Micromechanical validation of a mesomodel for plasticity in composites.

European Journal of Mechanics A - Solids, 60, 58-69. https://doi.org/10.1016/j.euromechsol.2016.06.008

\section{Important note}

To cite this publication, please use the final published version (if applicable).

Please check the document version above.

\section{Copyright}

Other than for strictly personal use, it is not permitted to download, forward or distribute the text or part of it, without the consent of the author(s) and/or copyright holder(s), unless the work is under an open content license such as Creative Commons.

\section{Takedown policy}

Please contact us and provide details if you believe this document breaches copyrights.

We will remove access to the work immediately and investigate your claim. 


\title{
Micromechanical validation of a mesomodel for plasticity in composites
}

\author{
Frans P. van der Meer* \\ Delft University of Technology, Faculty of Civil Engineering and Geosciences, PO Box 5048, 2600 GA Delft, The Netherlands
}

\begin{abstract}
In this paper, the performance of a recent homogenized orthotropic plasticity model for fiber reinforced composites (Vogler et al. (2013)) is investigated by comparing the model response against a micromechanical model. It is assumed that the micromechanical model which contains a recent plasticity model for polymers (Melro et al. (2013)) offers a realistic representation of the plastic deformation of composite materials. Under that assumption, the performance of the homogenized model can be assessed based on the question how well it reproduces micromechanical results. Improved consistent tangent formulations for both plasticity models are presented and a study into the representative volume element size for elasto-plastic micromechanical analysis is performed. Micromechanical simulations of basic load cases are used to generate input for the homogenized model. Next, the response of the two models is compared for a range of different load cases. The loss of accuracy due to necessary simplifications in the homogenized model is quantified in the comparison. These simplifications in the mesomodel include ignoring the stress in fiber direction, disregarding the influence stress orientation under combined longitudinal shear and transverse loading, and the use of a single constant plastic Poisson ratio.
\end{abstract}

Keywords: Composite laminates, micromechanics, homogenized model, plasticity

\section{Introduction}

Analysis of composite materials can be performed on different scales of observation. A popular scale for failure analysis is the mesoscale, where individual plies in a laminate are modeled as homogeneous orthotropic materials. On this scale, it is possible to distinguish between failure inside the plies and failure between the plies or delamination. More information is available on the microscale, where fibers and matrix are modeled separately. On the microlevel, it is possible to use constitutive models for the matrix, the fiber and the fiber/matrix interface that account for plasticity and damage, while mesomodels have to account for these processes in a homogenized manner. When it comes to linear elasticity, homogenization is very effective. Homogenized elasticity parameters can be extracted from simple micromechanical simulations and the resulting homogenized elasticity model is accurate for all linear deformations. However, when nonlinearities are present, formulating homogenized constitutive relations for the composite material is a challenge, where one has to find a balance between accuracy under general conditions and simplicity of the characterization. The model should be accurate for a wide range of loading scenarios but the required input should come from a limited set of basic tests.

An important example of where this challenge is manifest for mesolevel models is in the formulation of failure criteria. After many years of research, many different formulations are available to interpolate strength in general stress

*Corresponding author. Tel.: +31 152785918

Email address: f.p.vandermeer@tudelft.nl (Frans P. van der Meer) 
space from a limited set of basic strength values [1-5]. In recent years there is a movement towards using micromechanical models to generate failure envelopes [6-9]. The idea behind this trend is that the number of input parameters for a micromodel is limited because the behavior of the constituents is relatively simple, while the micromodel can be subjected to arbitrary stress states and histories.

Another ingredient for mesolevel failure analysis is a representation of shear nonlinearity. It has been shown that accounting for shear nonlinearity can make a significant difference on subcritical damage development, which in turn influences the final failure of a laminate $[10,11]$. For a laminate that is loaded in-plane, the most significant plastic deformations will occur in the in-plane shear strain component. In orthotropic elasticity there is no coupling between shear and axial deformations, which means that shear stress is a linear scalar relation of shear strain. For that reason it is possible to introduce nonlinear shear behavior by making this scalar relation a nonlinear relation without considering interaction with other stress and strain components. This allows for simple formulations for shear nonlinearity that have been used by different authors [10-13]. However, the underlying physical mechanism of plastic deformation of the polymer matrix is a continuum phenomenon which can only be described accurately in a full three-dimensional constitutive law. Plasticity of epoxy matrices is a pressure dependent nonlinear process [14]. Even though the fibers and loading in a laminate often ensure that the primary direction of plastic deformation is the in-plane shear direction, this process is never independent from hydrostatic pressure and transverse stresses that can be expected to be present.

Different formulations for orthotropic plasticity can be found in literature [15-17]. However, in case of hardening plasticity in composite materials it is crucial to make sure that there is no plasticity in the part of the homogenized response that represents the fibers. After all, the physical process that is represented by the homogenized plasticity model is plasticity in the matrix material and not in the, much stiffer, fibers. Recently, Vogler et al. [18] proposed a homogenized hardening plasticity model for orthotropic materials that is formulated in full three-dimensional space. The invariant-based formulation in this model excludes the influence of the composite stress in fiber direction on the plasticity and the corresponding flow rule prevents any plastic strain in fiber direction.

An alternative to homogenized constitutive methods can be found in multiscale analysis or $\mathrm{FE}^{2}$ [19]. Multiscale analysis connects to the idea that nonlinear processes in a material with a specific microstructure can be described accurately with a micromechanical numerical model without loss of generality. More basic constitutive models can be used for the different phases in the microstructure and the complexity of the composite material behavior follows from the micromechanics. In $\mathrm{FE}^{2}$ a finite element model on the higher scale is coupled in every integration point to a microlevel finite element model. Downside of this approach is that it is computationally very heavy. Therefore, good homogenized constitutive models will remain important. In order to be able to make a justified choice between the simplified homogenized approach and the expensive $\mathrm{FE}^{2}$ approach, the central question is to what extent the homogenized model can reproduce micromechanical results for arbitrary load cases.

In this paper, the performance of the plasticity model by Vogler et al. [18] is examined against micromechanical results. The micromodel is used to generate input for the mesomodel. Subsequently, the question is answered to what extent the mesomodel accurately represents the micromechanical response for non-calibrated cases. For matrix plasticity on the microlevel, the recent pressure dependent plasticity model by Melro et al. [20] is used. Minor changes to the formulation of the mesolevel and microlevel constitutive models are proposed. Furthermore, a study into the size of representative volume element (RVE) that is needed for micromechanical plasticity analysis is presented. This paper is purely concerned with plasticity. In complete analysis of composite materials, damage and fracture will at some point take place. For a pure assessment of the performance of the plasticity models, damage is excluded from both micro and mesolevel models in the current study. 


\section{Model formulation}

In this section, the plasticity models by Melro et al. [20] and Vogler et al. [18] are presented in slightly revised form. The aim is to be complete, so that the reader can reproduce the exact implementation used for the current investigation, without providing motivation for all the underlying choices. For more background on the constitutive models, the reader is referred to the original publications $[18,20]$. Differences with the original version of the models will be mentioned explicitly.

\subsection{Mathematical notations}

In this section, index notation and matrix notation will be used alternately. In index notation, summation over repeated indices is assumed. In matrix notation, boldfaced upper case roman symbols indicate second or fourth order tensors, while boldfaced lower case symbols indicate vectors. In matrix notation, the number of dots used to denote multiplication indicates the number of indices over which summation takes place, which implies that multiplication without dot indicates an outer product.

\subsection{Micromechanical model}

The micromechanical model consists of elastic isotropic fibers and the hardening plasticity model developed by Melro et al. [20,21]. With respect to the original formulation, changes are proposed to the formulation of the consistent tangent.

\subsubsection{Stress evaluation}

Stress evaluation for the matrix plasticity model begins with evaluation of the trial stress:

$$
\sigma_{i j, n}^{\mathrm{tr}}=D_{i j k l}\left(\varepsilon_{i j, n}-\varepsilon_{i j, n-1}^{\mathrm{p}}\right)
$$

where $\varepsilon_{i j, n}$ and $\sigma_{i j, n}^{\mathrm{tr}}$ are the strain and trial stress tensors at time $n, D_{i j k l}$ is the fourth order isotropic elasticity tensor and $\varepsilon_{i j, n-1}^{\mathrm{p}}$ is the plastic strain from the previous time step.

In tensor notation, the elasticity tensor is written as:

$$
D_{i j k l}=2 G \delta_{i j k l}^{\mathrm{s}}+\left(K-\frac{2}{3} G\right) \delta_{i j} \delta_{k l}
$$

where $G$ and $K$ are the shear and bulk modulus of the material, $\delta_{i j}$ is the Kronecker delta and $\delta_{i j k l}^{\mathrm{s}}$ is the symmetric version of the fourth order identity tensor, accounting for symmetry of the strain tensor:

$$
\delta_{i j k l}^{\mathrm{s}}=\frac{1}{2}\left(\delta_{i k} \delta_{j l}+\delta_{i l} \delta_{j k}\right)
$$

The yield function is given as:

$$
f\left(\sigma, \varepsilon_{\mathrm{eq}}^{\mathrm{p}}\right)=6 J_{2}(\sigma)+2 I_{1}(\sigma)\left(\sigma_{\mathrm{c}}\left(\varepsilon_{\mathrm{eq}}^{\mathrm{p}}\right)-\sigma_{\mathrm{t}}\left(\varepsilon_{\mathrm{eq}}^{\mathrm{p}}\right)\right)-2 \sigma_{\mathrm{c}}\left(\varepsilon_{\mathrm{eq}}^{\mathrm{p}}\right) \sigma_{\mathrm{t}}\left(\varepsilon_{\mathrm{eq}}^{\mathrm{p}}\right)=0
$$

where $J_{2}$ is the second deviatoric stress invariant and $I_{1}$ is the first stress invariant. The parameters $\sigma_{\mathrm{c}}$ and $\sigma_{\mathrm{t}}$ represent the yield stress in uniaxial compression and uniaxial tension. Both of these are not constants but rather a function of the equivalent plastic strain $\varepsilon_{\mathrm{eq}}^{\mathrm{p}}$, which is defined as the time integral of

$$
\dot{\varepsilon}_{\mathrm{eq}}^{\mathrm{p}}=\sqrt{k \dot{\boldsymbol{\varepsilon}}^{\mathrm{p}}: \dot{\varepsilon}^{\mathrm{p}}}
$$


where $k$ is related to the plastic Poisson ratio $v_{\mathrm{p}}$ as $k=1 /\left(1+2 v_{\mathrm{p}}\right)$. The expressions for $\sigma_{\mathrm{c}}$ and $\sigma_{\mathrm{t}}$ are piece-wise linear functions extracted from uniaxial stress-strain curves for a given $v_{\mathrm{p}}$.

If $f\left(\sigma_{n}^{\mathrm{tr}}, \varepsilon_{\mathrm{eq}, n-1}^{\mathrm{p}}\right)>0$, the plastic strain is increased to until

$$
f\left(\sigma_{n}, \varepsilon_{\mathrm{eq}, n}^{\mathrm{p}}\right)=0
$$

From here on, the subscript $n$ is dropped for notational simplicity. The increment in plastic strain is found in a return mapping algorithm where the nonlinear Eq. (6) is solved iteratively. Both unknowns $\sigma$ and $\varepsilon_{\mathrm{eq}}^{\mathrm{p}}$ are expressed in terms of the increment in plastic multiplier $\Delta \gamma$ to make Eq. (6) a function of a single scalar unknown. A non-associative flow rule is introduced by Melro et al. [20] that defines the increment in plastic strain with Euler backward time integration as:

$$
\Delta \boldsymbol{\varepsilon}^{\mathrm{p}}=\Delta \gamma\left(3 \mathbf{S}+\frac{2}{9} \alpha I_{1} \mathbf{I}\right)
$$

where $\mathbf{S}$ is the deviatoric stress tensor and $\mathbf{I}$ the identity matrix. Note that $\mathbf{S}$ and $I_{1}$ are expressed in terms of the stress tensor at the end of the return mapping procedure. The parameter $\alpha$ is related to the plastic Poisson ratio $v_{\mathrm{p}}$ :

$$
\alpha=\frac{9}{2} \frac{1-2 v_{\mathrm{p}}}{1+v_{\mathrm{p}}}
$$

With this flow rule, the relation between stress and $\Delta \gamma$ can be written as:

$$
\sigma=\frac{\mathbf{S}^{\mathrm{tr}}}{1+6 G \Delta \gamma}+\frac{\frac{1}{3} \mathrm{t}_{1}^{\mathrm{t} \mathbf{I}}}{1+2 K \alpha \Delta \gamma}=\frac{\mathbf{S}^{\mathrm{tr}}}{\zeta_{s}}+\frac{\frac{1}{3} I_{1}^{\mathrm{r} \mathbf{I}}}{\zeta_{p}}
$$

Note that $\mathbf{S}^{\mathrm{tr}}$ and $I_{1}^{\mathrm{tr}}$ in Eq. (9) are expressed in terms of trial stress and therefore constant during the return mapping procedure. The relation between the increment in equivalent plastic strain and the increment in plastic multiplier can be written as:

$$
\Delta \varepsilon_{\mathrm{eq}}^{\mathrm{p}}=\Delta \gamma \sqrt{k \mathbf{M}: \mathbf{M}}
$$

where $\mathbf{M}$ is the direction of plastic flow. In Eq. (7) the direction of plastic flow was expressed in terms of final stresses, alternatively it can be expressed in terms of trial stress and $\Delta \gamma$ :

$$
\mathbf{M}=\frac{3 \mathbf{S}^{\mathrm{tr}}}{\zeta_{s}}+\frac{\frac{2}{9} \alpha I_{1}^{\mathrm{t} \mathbf{I}}}{\zeta_{p}}
$$

With Eqs. (9) and (10), the yield function becomes a function of $\Delta \gamma$ only. This $f(\Delta \gamma)=0$ is solved iteratively. Because of the non-associative flow rule, it is possible that in exceptional cases there exists a solution for $\Delta \gamma<0$, which is thermodynamically inadmissible. The root is found with Newton's method starting at $\Delta \gamma=0$. If a negative root is obtained, this solution is discarded and another root is found in a less efficient algorithm that searches only in $\Delta \gamma>0$ direction.

For robustness and efficiency of the return mapping algorithm, exact linearization of $\partial f / \partial \Delta \gamma$ is needed. The linearization reads:

$$
\frac{\partial f}{\partial \Delta \gamma}=-\frac{72 G J_{2}^{\mathrm{tr}}}{\zeta_{s}^{3}}-\frac{4\left(\sigma_{\mathrm{c}}-\sigma_{\mathrm{t}}\right) K \alpha I_{1}^{\mathrm{rr}}}{\zeta_{p}^{2}}+\frac{\partial f}{\partial \varepsilon_{\mathrm{eq}}^{\mathrm{p}}} \frac{\partial \Delta \varepsilon_{e q}^{\mathrm{p}}}{\partial \Delta \gamma}
$$


The derivatives in the final term of Eq. (12) are given by:

$$
\begin{aligned}
& \frac{\partial f}{\partial \varepsilon_{\mathrm{eq}}^{\mathrm{p}}}=2\left(H_{\mathrm{c}}-H_{\mathrm{t}}\right) \frac{I_{1}^{\mathrm{tr}}}{\zeta_{p}}-2\left(\sigma_{\mathrm{c}} H_{\mathrm{t}}+\sigma_{\mathrm{t}} H_{\mathrm{c}}\right) \equiv \hat{H} \\
& \frac{\partial \varepsilon_{\mathrm{eq}}^{\mathrm{p}}}{\partial \Delta \gamma}=\sqrt{\frac{1}{1+2\left(v_{\mathrm{p}}\right)^{2}}}\left(\sqrt{A}-\frac{\Delta \gamma}{2 \sqrt{A}}\left(\frac{216 G J_{2}^{\mathrm{tr}}}{\zeta_{s}^{3}}+\frac{16 \alpha^{3} K\left(I_{1}^{\mathrm{tr}}\right)^{2}}{27 \zeta_{p}^{3}}\right)\right)
\end{aligned}
$$

where $H_{\mathrm{c}}$ and $H_{\mathrm{t}}$ are the hardening moduli defined as the derivatives of input functions $\sigma_{\mathrm{c}}$ and $\sigma_{\mathrm{t}}$ with respect to $\varepsilon_{\mathrm{eq}}^{\mathrm{p}}$, while $A$ is defined as:

$$
A=\frac{18 J_{2}^{\mathrm{tr}}}{\zeta_{s}^{2}}+\frac{4}{27}\left(\frac{\alpha I_{1}^{\mathrm{tr}}}{\zeta_{p}}\right)^{2}
$$

\subsubsection{Consistent linearization}

Taking the derivative of stress according to Eq. (9) with respect to strain gives:

$$
\frac{\partial \sigma_{i j}}{\partial \varepsilon_{k l}}=\frac{1}{\zeta_{s}} \frac{\partial S_{i j}^{\mathrm{tr}}}{\partial \varepsilon_{k l}}+\frac{\delta_{i j}}{3 \zeta_{p}} \frac{\partial I_{1}^{\mathrm{tr}}}{\partial \varepsilon_{k l}}-\frac{S_{i j}^{\mathrm{tr}}}{\zeta_{s}^{2}} 6 G \frac{\partial \Delta \gamma}{\partial \varepsilon_{k l}}-\frac{I_{1}^{\mathrm{tr}} \delta_{i j}}{3 \zeta_{p}^{2}} 2 K \alpha \frac{\partial \Delta \gamma}{\partial \varepsilon_{k l}}
$$

In order to expand this expression, a relation between the rate in plastic multiplier and the rate in strain is needed. However, because the plastic multiplier is computed in an iterative procedure, there is no explicit relation from which the derivative $\partial \Delta \gamma / \partial \varepsilon$ can be evaluated. Instead, use is made of the consistency condition, which states that the condition $\dot{f}=0$ must hold from one iteration to the next, because $f$ remains equal to zero. If $f$ is written as a function of independent unknowns $\boldsymbol{\varepsilon}$ and $\Delta \gamma$, one obtains:

$$
\delta f=\frac{\partial f}{\partial \boldsymbol{\varepsilon}}: \delta \boldsymbol{\varepsilon}+\frac{\partial f}{\partial \Delta \gamma} \delta \Delta \gamma=0 \Rightarrow \frac{\partial \Delta \gamma}{\partial \boldsymbol{\varepsilon}}=\frac{1}{\eta} \frac{\partial f}{\partial \boldsymbol{\varepsilon}}
$$

with

$$
\eta=-\frac{\partial f}{\partial \Delta \gamma}
$$

The expression for $\partial f / \partial \Delta \gamma$ is already given in Eq. (12). The derivative $\partial f / \partial \boldsymbol{\varepsilon}$ is expressed as:

$$
\frac{\partial f}{\partial \boldsymbol{\varepsilon}}=\frac{6}{\zeta_{s}^{2}} \frac{\partial J_{2}^{\mathrm{tr}}}{\partial \boldsymbol{\varepsilon}}+\frac{2\left(\sigma_{\mathrm{c}}-\sigma_{\mathrm{t}}\right)}{\zeta_{p}} \frac{\partial I_{1}^{\mathrm{tr}}}{\partial \boldsymbol{\varepsilon}}+\frac{\partial f}{\partial \varepsilon_{\mathrm{eq}}^{\mathrm{p}}} \frac{\partial \varepsilon_{\mathrm{eq}}^{\mathrm{p}}}{\partial \boldsymbol{\varepsilon}}
$$

with

$$
\begin{aligned}
\frac{\partial J_{2}^{\mathrm{tr}}}{\partial \varepsilon_{k l}} & =S_{i j}^{\mathrm{tr}} \frac{\partial S_{i j}^{\mathrm{tr}}}{\partial \varepsilon_{k l}}=2 G S_{i j}^{\mathrm{tr}} \delta_{i j k l}^{s}-\frac{2}{3} G S_{i j}^{\mathrm{tr}} \delta_{i j} \delta_{k l}=2 G S_{k l}^{\mathrm{tr}} \\
\frac{\partial I_{1}^{\mathrm{tr}}}{\partial \varepsilon_{k l}} & =3 K \delta_{k l}
\end{aligned}
$$

The final term in Eq. (19), which originates from the dependence of $\sigma_{\mathrm{c}}$ and $\sigma_{\mathrm{t}}$ on $\varepsilon$ through the presence of the trial stress in Eqs. (10) and (11), was ignored by Melro et al. [20]. Nevertheless, is was found to be relevant for good convergence in the present work. The final term is completed with the following expressions:

$$
\frac{\partial \varepsilon_{\mathrm{eq}}^{\mathrm{p}}}{\partial \varepsilon_{i j}}=\frac{(\Delta \gamma)^{2} k}{\Delta \varepsilon_{\mathrm{eq}}^{\mathrm{p}}} M_{k l} \frac{\partial M_{k l}}{\partial \varepsilon_{i j}} \equiv E_{i j}
$$


with

$$
\frac{\partial M_{i j}}{\partial \varepsilon_{k l}}=\frac{6 G\left(\delta_{i j k l}^{\mathrm{s}}-\frac{1}{3} \delta_{i j} \delta_{k l}\right)}{\zeta_{s}}+\frac{\frac{2}{3} \alpha K \delta_{i j} \delta_{k l}}{\zeta_{p}}
$$

Substituting relations from Eqs. (17), (20) and (21) into Eq. (16) gives:

$$
\begin{aligned}
\frac{\partial \sigma_{i j}}{\partial \varepsilon_{k l}}= & \frac{2 G}{\zeta_{s}}\left(\delta_{i j k l}^{s}-\frac{1}{3} \delta_{i j} \delta_{k l}\right)+\frac{K}{\zeta_{p}} \delta_{i j} \delta_{k l} \\
& -\frac{72 G^{2} S_{i j}^{\operatorname{tr}} S_{k l}^{\operatorname{tr}}}{\eta \zeta_{s}^{4}}-\frac{36 K G\left(\sigma_{c}-\sigma_{t}\right) S_{i j}^{\mathrm{tr}} \delta_{k l}}{\eta \zeta_{s}^{2} \zeta_{p}} \\
& -\frac{8 K G \alpha I_{1}^{\mathrm{tr}} \delta_{i j} S_{k l}^{\mathrm{tr}}}{\eta \zeta_{s}^{2} \zeta_{p}^{2}}-\frac{4 K^{2} \alpha I_{1}^{\mathrm{tr}}\left(\sigma_{c}-\sigma_{t}\right) \delta_{i j} \delta_{k l}}{\eta \zeta_{p}^{3}} \\
& -\frac{6 G S_{i j}^{\mathrm{tr}} E_{k l} \hat{H}}{\eta \zeta_{s}^{2}}-\frac{2 K \alpha I_{1}^{\mathrm{tr}} \delta_{i j} E_{k l} \hat{H}}{3 \eta \zeta_{p}^{2}}
\end{aligned}
$$

where $E_{k l}$ is the shorthand notation for the tensor introduced in Eq. (22) and $\hat{H}$ for the derivative from Eq. (13). The consistent tangent can be reordered to an expression in the notation adopted by Melro et al. [20]:

$$
\frac{\partial \boldsymbol{\sigma}}{\partial \boldsymbol{\varepsilon}}=\beta \mathbf{I}_{4}^{\mathrm{s}}+\left(\phi-\frac{\beta}{3}\right) \mathbf{I I}-\rho \mathbf{S}^{\mathrm{tr}} \mathbf{I}-\chi \mathbf{S}^{\operatorname{tr}} \mathbf{S}^{\operatorname{tr}}-\psi \mathbf{I} \mathbf{S}^{\operatorname{tr}}-\omega \mathbf{S}^{\operatorname{tr}} \mathbf{E}-\xi \mathbf{I} \mathbf{E}
$$

where $\mathbf{I}_{4}^{\mathrm{s}}$ is the fourth order tensor from Eq. (3) and the different coefficients are defined as:

$$
\begin{aligned}
\beta & =\frac{2 G}{\zeta_{s}} \\
\phi & =\frac{K}{\zeta_{p}}-\frac{4 K^{2} \alpha I_{1}^{\mathrm{tr}}\left(\sigma_{\mathrm{c}}-\sigma_{\mathrm{t}}\right)}{\eta \zeta_{p}^{3}} \\
\rho & =\frac{36 K G\left(\sigma_{\mathrm{c}}-\sigma_{\mathrm{t}}\right)}{\eta \zeta_{s}^{2} \zeta_{p}} \\
\chi & =\frac{72 G^{2}}{\eta \zeta_{s}^{4}} \\
\psi & =\frac{8 K G \alpha I_{1}^{\mathrm{tr}}}{\eta \zeta_{s}^{2} \zeta_{p}^{2}} \\
\omega & =\frac{6 G \hat{H}}{\eta \zeta_{s}^{2}} \\
\xi & =\frac{2 K \alpha I_{1}^{\mathrm{tr}} \hat{H}}{3 \eta \zeta_{p}^{2}}
\end{aligned}
$$

Note that for $\phi$ the order of $\zeta_{s}$ in the denominator is one order higher than in the original paper. Furthermore, the terms with $\mathbf{E}$ are new. These are related to the dependence of $\Delta \varepsilon_{\mathrm{eq}}^{\mathrm{p}}$ on $\sigma^{\mathrm{tr}}$.

\subsection{Mesomechanical model}

For the mesomechanical model, the constitutive model by Vogler et al. [18] is used. Also in this model small changes are proposed and therefore the formulation is briefly presented here. 


\subsubsection{Stress evaluation}

Vogler et al. [18] have derived their yield function from a set of stress invariants. In the end of the presentation of the model, they state that the yield function can be written down in simplified form and this formulation will be followed here. All expressions below are given in local coordinate frame with the first stress component in fiber direction. In a finite element implementation the evaluation of the constitutive law is preceded by a transformation of strain to the local frame and followed by a transformation of stress and stiffness to the global frame.

The yield function is written in Voigt notation as:

$$
f=\frac{1}{2} \boldsymbol{\sigma} \cdot \mathbf{A} \cdot \boldsymbol{\sigma}+\mathbf{a} \cdot \boldsymbol{\sigma}-1
$$

with

$$
\mathbf{A}=\left[\begin{array}{cccccc}
0 & 0 & 0 & 0 & 0 & 0 \\
0 & \frac{1}{2} \alpha_{1}+2 \alpha_{32} & -\frac{1}{2} \alpha_{1}+2 \alpha_{32} & 0 & 0 & 0 \\
0 & -\frac{1}{2} \alpha_{1}+2 \alpha_{32} & \frac{1}{2} \alpha_{1}+2 \alpha_{32} & 0 & 0 & 0 \\
0 & 0 & 0 & 2 \alpha_{1} & 0 & 0 \\
0 & 0 & 0 & 0 & 2 \alpha_{2} & 0 \\
0 & 0 & 0 & 0 & 0 & 2 \alpha_{2}
\end{array}\right]
$$

and

$$
\mathbf{a}=\left\{\begin{array}{llllll}
0 & \alpha_{3} & \alpha_{3} & 0 & 0 & 0
\end{array}\right\}^{T}
$$

The $\alpha_{i}$ coefficients are piecewise linear functions of equivalent plastic strain $\varepsilon_{\mathrm{eq}}^{\mathrm{p}}$ obtained from stress-strain curves from basic cases. For $\alpha_{32}$ and $\alpha_{3}$ there are two versions, one for compression and one for tension. The decision of which of these two versions is used depends on the sign of $\sigma_{2}+\sigma_{3}$. Continuity in $f\left(\sigma, \varepsilon_{\mathrm{eq}}^{\mathrm{p}}\right)$ is ensured by the fact that both $\alpha_{32}$ and $\alpha_{3}$ do not contribute to $f$ when $\sigma_{2}+\sigma_{3}=0$.

Stress update is based on:

$$
\sigma=\sigma^{\mathrm{tr}}-\mathbf{D}^{\mathrm{e}} \cdot \Delta \boldsymbol{\varepsilon}^{\mathrm{p}}
$$

where $\mathbf{D}^{\mathrm{e}}$ is the orthotropic elasticity matrix and

$$
\Delta \varepsilon^{\mathrm{p}}=\Delta \gamma \mathbf{n}_{g}
$$

where $\mathbf{n}_{g}$ is the derivative of the flow potential with respect to stress defined as:

$$
\mathbf{n}_{g}=\mathbf{B} \cdot \boldsymbol{\sigma}
$$

where $\mathbf{B}$ has the same shape as $\mathbf{A}$ in Eq. (34), except that constants $\beta_{i}$ replace the variables $\alpha_{i}$. Substitution of (37) and (38) into (36) gives:

$$
\boldsymbol{\sigma}=\left[\mathbf{I}+\Delta \gamma \mathbf{D}^{\mathrm{e}} \cdot \mathbf{B}\right]^{-1} \cdot \boldsymbol{\sigma}^{\mathrm{tr}} \equiv \mathbf{F} \cdot \boldsymbol{\sigma}^{\mathrm{tr}}
$$

With this expression, $\sigma$ becomes a function of a single unknown, $\Delta \gamma$. The yield function can be written as a function of this one unknown after the dependence of the $\alpha_{i}$ variables on $\Delta \gamma$ through the equivalent plastic strain is taken into account. The equivalent plastic strain rate is defined in Voigt notation with engineering strain as:

$$
\dot{\varepsilon}_{\mathrm{eq}}^{\mathrm{p}}=\sqrt{\dot{\boldsymbol{\varepsilon}}^{\mathrm{p}} \cdot \mathbf{J} \cdot \dot{\boldsymbol{\varepsilon}}^{\mathrm{p}}} \equiv\left\|\dot{\boldsymbol{\varepsilon}}^{\mathrm{p}}\right\|_{\mathbf{J}}
$$


with

$$
\mathbf{J}=\operatorname{diag}\left\{\frac{1}{2}, \frac{1}{2}, \frac{1}{2}, \frac{1}{4}, \frac{1}{4}, \frac{1}{4}\right\}
$$

Note that the difference in shear and axial terms in the $\mathbf{J}$ matrix is a consequence of the use of Voigt notation and engineering strain. The unconventional norm $\left\|\dot{\boldsymbol{\varepsilon}}^{\mathrm{p}}\right\|_{\mathbf{J}}$ is equivalent to the tensor notation expression used in the original paper $\sqrt{\frac{1}{2} \dot{\varepsilon}_{i j}^{\mathrm{p}} \dot{\varepsilon}_{i j}^{\mathrm{p}}}$. Euler backward time integration of the evolution of $\varepsilon_{\mathrm{eq}}^{\mathrm{p}}$ gives:

$$
\Delta \varepsilon_{\mathrm{eq}}^{\mathrm{p}}=\Delta \gamma\left\|\mathbf{n}_{g}\right\|_{\mathbf{J}}
$$

Now, the yield function is a function with only one unknown, $\Delta \gamma$. The value of $\Delta \gamma$ for which $f=0$ is found iteratively with Netwon's method. This procedure requires an expression for the derivative $\partial f / \partial \Delta \gamma$. For robustness and efficiency of the iterative procedure, care is required to include all dependencies in this linearization. Here, $f$ is first considered a function of independent unknowns $\sigma$ and $\varepsilon_{\mathrm{eq}}^{\mathrm{p}}$ and subsequently the chain rule is applied to account for dependence of both of these on $\Delta \gamma$ :

$$
\frac{\partial f(\Delta \gamma)}{\partial \Delta \gamma}=\frac{\partial f\left(\sigma, \varepsilon_{\mathrm{eq}}^{\mathrm{p}}\right)}{\partial \sigma} \cdot \frac{\partial \sigma}{\partial \Delta \gamma}+\frac{\partial f\left(\sigma, \varepsilon_{\mathrm{eq}}^{\mathrm{p}}\right)}{\partial \varepsilon_{\mathrm{eq}}^{\mathrm{p}}} \frac{\partial \varepsilon_{\mathrm{eq}}^{\mathrm{p}}(\Delta \gamma)}{\partial \Delta \gamma}
$$

with

$$
\begin{aligned}
& \frac{\partial f\left(\boldsymbol{\sigma}, \varepsilon_{\mathrm{eq}}^{\mathrm{p}}\right)}{\partial \boldsymbol{\sigma}}=\mathbf{A} \cdot \boldsymbol{\sigma}+\mathbf{a} \equiv \mathbf{n}_{f} \\
& \frac{\partial f\left(\boldsymbol{\sigma}, \varepsilon_{\mathrm{eq}}^{\mathrm{p}}\right)}{\partial \varepsilon_{\mathrm{eq}}^{\mathrm{p}}}=\frac{1}{2} \boldsymbol{\sigma} \cdot \frac{\partial \mathbf{A}}{\partial \varepsilon_{\mathrm{eq}}^{\mathrm{p}}} \cdot \boldsymbol{\sigma}+\frac{\partial \mathbf{a}}{\partial \varepsilon_{\mathrm{eq}}^{\mathrm{p}}} \cdot \boldsymbol{\sigma}
\end{aligned}
$$

The derivatives of $\mathbf{A}$ and $\mathbf{a}$ with respect to $\varepsilon_{\mathrm{eq}}^{\mathrm{p}}$ follow from the hardening functions for the coefficients $\alpha_{i}$ which directly depend on $\varepsilon_{\text {eq }}^{\mathrm{p}}$. The derivative of stress with respect to $\Delta \gamma$ is obtained from Eq. (36). Taking the derivative of Eq. (36) after substitution of Eqs. (37) and (38) gives:

$$
\frac{\partial \sigma}{\partial \Delta \gamma}=-\Delta \gamma \mathbf{D}^{\mathrm{e}} \cdot \mathbf{B} \cdot \frac{\partial \sigma}{\partial \Delta \gamma}+\mathbf{D}^{\mathrm{e}} \cdot \mathbf{B} \cdot \boldsymbol{\sigma} \Rightarrow \frac{\partial \boldsymbol{\sigma}}{\partial \Delta \gamma}=-\mathbf{F} \cdot \mathbf{D}^{\mathrm{e}} \cdot \mathbf{B} \cdot \boldsymbol{\sigma}
$$

The derivative of $\varepsilon_{\mathrm{eq}}^{\mathrm{p}}$ with respect to $\Delta \gamma$ is given by:

$$
\frac{\partial \varepsilon_{\mathrm{eq}}^{\mathrm{p}}(\Delta \gamma)}{\partial \Delta \gamma}=\frac{\partial \varepsilon_{\mathrm{eq}}^{\mathrm{p}}(\sigma, \Delta \gamma)}{\partial \Delta \gamma}+\frac{\partial \varepsilon_{\mathrm{eq}}^{\mathrm{p}}(\sigma, \Delta \gamma)}{\partial \sigma} \cdot \frac{\partial \sigma}{\partial \Delta \gamma}
$$

with

$$
\frac{\partial \varepsilon_{\mathrm{eq}}^{\mathrm{p}}(\sigma, \Delta \gamma)}{\partial \Delta \gamma}=\left\|\mathbf{n}_{g}\right\|_{\mathbf{J}}
$$

and

$$
\frac{\partial \varepsilon_{\mathrm{eq}}^{\mathrm{p}}(\boldsymbol{\sigma}, \Delta \gamma)}{\partial \boldsymbol{\sigma}}=-\Delta \gamma \frac{\mathbf{J} \cdot \mathbf{n}_{g}}{\left\|\mathbf{n}_{g}\right\|_{\mathbf{J}}} \cdot \mathbf{B}
$$




\subsubsection{Characterization}

The constitutive law is characterized by filling the parameters $\alpha_{i}$ in the yield function (Eq. (33)) and parameters $\beta_{i}$ in the flow rule (Eq. (38)). Relations between the $\alpha_{i}$ values and the stress values measured from basic tests are:

$$
\begin{aligned}
& \alpha_{1}=\frac{1}{Y_{\mathrm{TS}}^{2}} \\
& \alpha_{2}=\frac{1}{Y_{\mathrm{LS}}^{2}} \\
& \alpha_{32}^{\mathrm{t}}=\frac{1-\frac{Y_{\mathrm{UT}}}{2 Y_{\mathrm{BT}}}-\alpha_{1} \frac{Y_{\mathrm{UT}}^{2}}{4}}{Y_{\mathrm{UT}}^{2}-2 Y_{\mathrm{BT}} Y_{\mathrm{UT}}} \\
& \alpha_{3}^{\mathrm{t}}=\frac{1}{2 Y_{\mathrm{BT}}}-2 \alpha_{32}^{\mathrm{t}} Y_{\mathrm{BT}} \\
& \alpha_{32}^{\mathrm{c}}=\frac{1-\frac{Y_{\mathrm{UC}}}{2 Y_{\mathrm{BC}}}-\alpha_{1} \frac{Y_{\mathrm{UC}}^{2}}{4}}{Y_{\mathrm{UC}}^{2}-2 Y_{\mathrm{BC}} Y_{\mathrm{UC}}} \\
& \alpha_{3}^{\mathrm{c}}=\frac{1}{2 Y_{\mathrm{BC}}}-2 \alpha_{32}^{\mathrm{c}} Y_{\mathrm{BC}}
\end{aligned}
$$

where $Y_{\mathrm{TS}}$ refers to transverse shear stress, $Y_{\mathrm{LS}}$ to longitudinal shear stress, $Y_{\mathrm{UT}}$ to uniaxial tensile stress, $Y_{\mathrm{BT}}$ to biaxial tensile stress, $Y_{\mathrm{UC}}$ to uniaxial compressive stress and $Y_{\mathrm{BC}}$ to biaxial compressive stress. All $Y_{\text {.. stress histories }}$ are expressed as a function of equivalent plastic strain. The computation of equivalent plastic strain histories from stress and strain histories will be discussed in Section 3.2.

Regarding the plastic potential and the $\beta_{i}$ parameters, a constant plastic Poisson ratio is assumed to exist. For a given value of $v_{\mathrm{p}}$, the ratio between $\beta_{1}$ and $\beta_{32}$ is given as:

$$
\beta_{32}=\frac{1-v_{\mathrm{p}}}{4\left(1+v_{\mathrm{p}}\right)} \beta_{1}
$$

The ratio between $\beta_{1}$ and $\beta_{2}$ has to be determined from a test with combines transverse and longitudinal shear. In the present work, the two parameters are assumed to be equal. Then the B-matrix is known apart from a constant factor, which will be canceled by the plastic multiplier $\gamma$. Setting $\beta_{1}=1+v_{\mathrm{p}}$ results in the following simple notation for the B-matrix:

$$
\mathbf{B}=\left[\begin{array}{cccccc}
0 & 0 & 0 & 0 & 0 & 0 \\
0 & 1 & -v_{\mathrm{p}} & 0 & 0 & 0 \\
0 & -v_{\mathrm{p}} & 1 & 0 & 0 & 0 \\
0 & 0 & 0 & 2\left(1+v_{\mathrm{p}}\right) & 0 & 0 \\
0 & 0 & 0 & 0 & 2\left(1+v_{\mathrm{p}}\right) & 0 \\
0 & 0 & 0 & 0 & 0 & 2\left(1+v_{\mathrm{p}}\right)
\end{array}\right]
$$

Note that the optimization procedure described by Vogler et al. [18] is not present in this implementation. The concept followed here is that measurement of strain in unloaded direction in a uniaxial test allows to estimate $v_{\mathrm{p}}$ directly from test data. With the additional assumption $\beta_{2}=\beta_{1}, v_{\mathrm{p}}$ completely defines the $\mathbf{B}$-matrix.

\subsubsection{Consistent linearization}

For deriving the consistent tangent, the procedure by Van der Meer and Sluys [17, 22] for a similar orthotropic plasticity model is followed. The variation in stress is expressed in terms of independent variations in strain and plastic 
multiplier as:

$$
\delta \boldsymbol{\sigma}=\mathbf{H} \cdot \delta \boldsymbol{\varepsilon}-\mathbf{H} \cdot \mathbf{n}_{g} \delta \Delta \gamma
$$

with $\mathbf{H}=\mathbf{F} \cdot \mathbf{D}^{\mathrm{e}}$. With the consistency condition $\delta f=0$, it is possible to eliminate $\delta \Delta \gamma$ from this relation. The dependence of $\varepsilon_{\mathrm{eq}}^{\mathrm{p}}$ on $\boldsymbol{\sigma}$ through $\mathbf{n}_{g}$ has to be accounted for:

$$
\begin{aligned}
\delta f & =\frac{\partial f\left(\sigma, \varepsilon_{\mathrm{eq}}^{\mathrm{p}}\right)}{\partial \sigma} \cdot \delta \sigma+\frac{\partial f\left(\sigma, \varepsilon_{\mathrm{eq}}^{\mathrm{p}}\right)}{\partial \varepsilon_{\mathrm{eq}}^{\mathrm{p}}}\left(\frac{\partial \varepsilon_{\mathrm{eq}}^{\mathrm{p}}(\sigma, \Delta \gamma)}{\partial \Delta \gamma} \delta \Delta \gamma+\frac{\partial \varepsilon_{\mathrm{eq}}^{\mathrm{p}}(\sigma, \Delta \gamma)}{\partial \sigma} \cdot \delta \sigma\right) \\
& =\left(\mathbf{n}_{f}+\frac{\partial f\left(\sigma, \varepsilon_{\mathrm{eq}}^{\mathrm{p}}\right)}{\partial \varepsilon_{\mathrm{eq}}^{\mathrm{p}}} \frac{\partial \varepsilon_{\mathrm{eq}}^{\mathrm{p}}(\sigma, \Delta \gamma)}{\partial \sigma}\right) \cdot \delta \boldsymbol{\sigma}+\frac{\partial f\left(\sigma, \varepsilon_{\mathrm{eq}}^{\mathrm{p}}\right)}{\partial \varepsilon_{\mathrm{eq}}^{\mathrm{p}}} \frac{\partial \varepsilon_{\mathrm{eq}}^{\mathrm{p}}(\sigma, \Delta \gamma)}{\partial \Delta \gamma} \delta \Delta \gamma
\end{aligned}
$$

Equating the variation in $f$ to zero gives:

$$
\delta \Delta \gamma=\frac{1}{\lambda} \overline{\mathbf{n}}_{f} \cdot \delta \sigma
$$

with

$$
\overline{\mathbf{n}}_{f}=\frac{\partial f(\sigma, \Delta \gamma)}{\partial \sigma}=\mathbf{n}_{f}+\frac{\partial f\left(\sigma, \varepsilon_{\mathrm{eq}}^{\mathrm{p}}\right)}{\partial \varepsilon_{\mathrm{eq}}^{\mathrm{p}}} \frac{\partial \varepsilon_{\mathrm{eq}}^{\mathrm{p}}(\sigma, \Delta \gamma)}{\partial \sigma}
$$

and

$$
\lambda=-\frac{\partial f\left(\sigma, \varepsilon_{\mathrm{eq}}^{\mathrm{p}}\right)}{\partial \varepsilon_{\mathrm{eq}}^{\mathrm{p}}} \frac{\partial \varepsilon_{\mathrm{eq}}^{\mathrm{p}}(\sigma, \Delta \gamma)}{\partial \Delta \gamma}
$$

Substitution of Eq. (60) into (58) gives:

$$
\delta \boldsymbol{\sigma}=\mathbf{H} \cdot \delta \boldsymbol{\varepsilon}-\frac{1}{\lambda} \mathbf{H} \cdot \mathbf{n}_{g} \overline{\mathbf{n}}_{f} \cdot \delta \boldsymbol{\sigma}
$$

or

$$
\delta \boldsymbol{\varepsilon}=\left[\mathbf{H}^{-1}+\frac{1}{\lambda} \mathbf{n}_{g} \overline{\mathbf{n}}_{f}\right] \cdot \delta \boldsymbol{\sigma}
$$

This relation is inverted with the Sherman-Morrison formula to give:

$$
\delta \boldsymbol{\sigma}=\left[\mathbf{H}-\frac{\mathbf{H} \cdot \mathbf{n}_{g} \overline{\mathbf{n}}_{f} \cdot \mathbf{H}}{\lambda+\overline{\mathbf{n}}_{f} \cdot \mathbf{H} \cdot \mathbf{n}_{g}}\right] \delta \boldsymbol{\varepsilon}
$$

Note that the difference with the tangent formulation by Vogler et al. [18] lies in the usage of $\overline{\mathbf{n}}_{f}$ from Eq. (61) instead of $\mathbf{n}_{f}$ from Eq. (44). Furthermore, a different expression for $\lambda$ is used: Vogler et al. [18] propose to use the derivative from Eq. (43) which includes dependency of $\sigma$ on $\Delta \gamma$. This dependency only exists for constant trial stress inside the return mapping algorithm. In Eq. (59), which is where $\lambda$ originates from, $\delta \sigma$ and $\delta \Delta \gamma$ are independent.

\section{Results}

The fundamental input for this study is the input used for the micromodel. All input for the mesomodel is extracted from micromodel results. In the micromodel, the fibers are represented as isotropic elastic continuum with Young's modulus $74000 \mathrm{MPa}$ and Poisson's ratio 0.2. For elastic behavior of the matrix, a Young's modulus of 3760 and Poisson's ratio of 0.3 are used. For plasticity, a plastic Poisson ratio of 0.39 is used and the fundamental hardening curves are given in Figure 1. The input data is based on that given for epoxy by Melro [21] except that the secondary hardening tail given for the compressive behavior is removed. In the current plasticity-only investigation, the combination of a second rising branch in the compression hardening curve with a plateau for the tension hardening curve leads to softening in tension for large strain values. In real life, failure would take place before such strain values are reached, but in the current investigation of purely plastic behavior, these strain values will occur. 


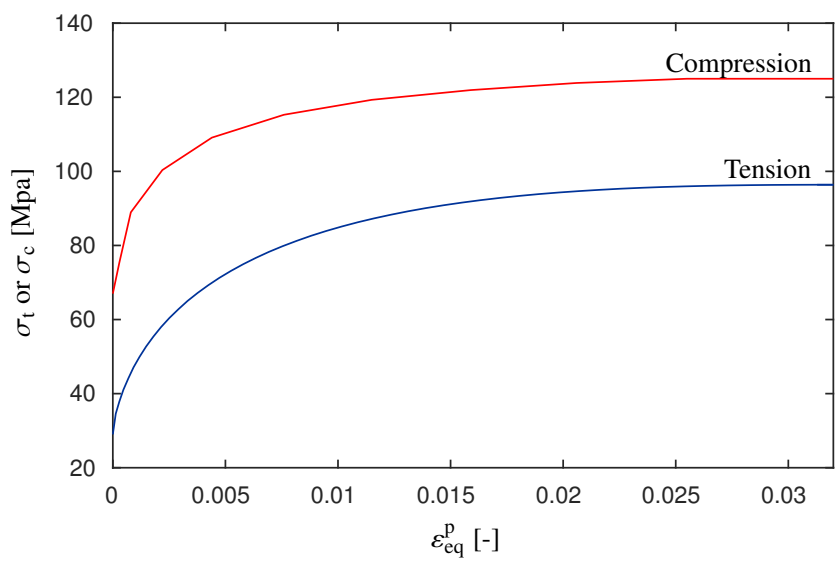

Figure 1: Input hardening curves for micromechanical plasticity model.

\subsection{RVE size study}

Before the comparison between the micro and mesolevel models can be done, the geometry of the micromodel must be defined. A representative volume element (RVE) with a random fiber distribution is used to simulate the composite response. First, the size of the RVE should be determined. The micromodel is only representative if increasing its size does not affect its averaged response.

Melro et al. [23] have investigated the influence of the size of the micromodel on the elastic response. Gonzalez et al. [24] have shown that for transverse compression the results from an elasto/plastic RVE of 70 fibers is similar the averaged response from different RVEs with 30 fibers each. Canal et al. [25] found that the statistical variation for transverse tension and shear for different RVEs of 30 fibers to be limited. In this section, a statistical study into the influence of RVE size on the elasto/plastic response for transverse tension and longitudinal shear is presented with a large number of different RVEs of different sizes. Random fiber distributions are generated with the discrete element simulation package HADES. Initial conditions for these simulations consist of a specified number of equally spaced discs in two dimensional space with pseudo-random velocities. The discs, representing the fibers, are located in two-dimensional periodic box which shrinks over time. A contact model with predefined minimum spacing between the fibers ensures that fibers do not overlap as the fiber density increases. When the desired fiber volume fraction is reached, the discrete element simulation is ended and the resulting geometry is meshed with Gmsh[26]. For threedimensional simulations, the mesh of three-node triangles is extruded to generate a single layer of six-node wedge elements. This means that a full three-dimensional deformation state can be represented. However, the single layer of elements in implies that strain does not vary in fiber direction and that all fibers are assumed to be perfectly straight and aligned.

For the mesh generation, a finite minimum distance between the fibers is needed, although in reality it is possible that fibers are in immediate contact. Geometric parameters for the generation of fiber distributions are a fiber diameter of $5 \mu \mathrm{m}$, fiber volume fraction of 0.6 and minimum spacing of $0.2 \mu \mathrm{m}$. Meshes are generated with typical element length of $0.5 \mu \mathrm{m}$ in the matrix, coarsening up to $2 \mu \mathrm{m}$ in the center of the fibers.

At first, two-dimensional plane strain simulations of uniaxial tension are performed. The micromodel is subjected to periodic boundary conditions. The mesh itself is also periodic, which means that periodic boundary conditions can be applied on the micromodel with linear constraints between degrees of freedom. The relative displacement between two matching nodes on opposite faces is constrained to be equal to the relative displacement of a master node with 
respect to the origin:

$$
\begin{aligned}
& \mathbf{u}(\Delta x, y, z)=\mathbf{u}(0, y, z)+\mathbf{u}(\Delta x, 0,0)-\mathbf{u}(0,0,0) \\
& \mathbf{u}(x, \Delta y, z)=\mathbf{u}(x, 0, z)+\mathbf{u}(0, \Delta y, 0)-\mathbf{u}(0,0,0) \\
& \mathbf{u}(x, y, \Delta z)=\mathbf{u}(x, y, 0)+\mathbf{u}(0,0, \Delta z)-\mathbf{u}(0,0,0)
\end{aligned}
$$

where $\mathbf{u}(0,0,0)$ is prescribed to zero to eliminate rigid translations and $\mathbf{u}(\Delta x, 0,0), \mathbf{u}(0, \Delta y, 0)$ and $\mathbf{u}(0,0, \Delta z)$ are three master corner nodes. Averaged deformations can be defined by prescribing the displacements of these master nodes. Averaged stresses (including zero stress condition) can be applied by keeping associated master node displacements unconstrained and applying an external force (or zero external force) on the master node. The periodic boundary constraints are equivalent to those described by Melro et al. [23], except for the option to prescribe certain averaged stress components instead of strain components, which is an option that was not given in [23]. The special constraints that exist along edges and in corners as given by Melro et al. [23] are not explicitly prescribed but automatically found when resolving newly added constraints to prevent a single degree of freedom to be slave and master at the same time. The resolved constraint for the corner node opposite to the origin, for instance, becomes:

$$
\mathbf{u}(\Delta x, \Delta y, \Delta z)=\mathbf{u}(\Delta x, 0,0)+\mathbf{u}(0, \Delta y, 0)+\mathbf{u}(0,0, \Delta z)-2 \mathbf{u}(0,0,0)
$$

For the final formulation of the constraints, it does not matter in which order the three lines of Eq. (66) are applied.

Averaged stress values are obtained through division of the sum of nodal forces along one of the faces over by area associated with that face. The averaged deformation gradient is obtained from the displacements of the three master corner nodes divided by the length of the unit cell in the associated direction. Strain is defined as the symmetric part of the deformation gradient. For shear strains engineering strain values are used.

In Figure 2 the stress-strain response for 50 different fiber distributions with micromodels consisting of 25 fibers are plotted. It is observed that there is much more scatter in the plastic response than there is in the initial stiffness. The response approaches a perfectly plastic response, although the curve remains slightly increasing because no complete plastic mechanism is found. The final distribution of equivalent plastic strain is visualized for three different fiber distributions in Figure 3. The exact strain field depends strongly on the particular fiber distribution. Plastic strain tends to localize under an angle between 45 and 90 degrees with the load direction. Periodic boundary conditions do not support a single localization band under an arbitrary angle. For localization under perfectly plastic conditions, the influence of this artificial constraint on the global response is expected to remain limited.

The fact that there is significant scatter in the micromodel response can be taken to mean that a single model of 25 fibers is not big enough to be statistically representative. The same procedure is repeated for different micromodel sizes. For each of a different number of fibers $n_{\mathrm{f}}$ with $\sqrt{n_{\mathrm{f}}} \in\{2,3, \ldots, 9\}, 50$ different micromodels are generated and subjected to uniaxial transverse tension with plane strain in fiber direction. A unit cell model with single fiber centered in the domain is also included. For each micromodel size, the mean and variation in stress level at a strain of 0.03 is plotted in Figure 4. The unit cell gives significantly higher final stress level than the larger micromodels. For increasing micromodel size, the mean response converges to stable value, while the amount of scatter decreases very slowly. The fact that there is still significant scatter in the response from the largest micromodels means that the response of any given micromodel of that size not yet truly representative. Error bars show the standard deviation and the shaded area indicates the $95 \%$ interval of confidence for the mean stress based on the data from the micromodels with $9 \times 9$ fibers. Variations in the mean are statistically insignificant from a micromodel size of $5 \times 5$ fibers.

The exercise is repeated for longitudinal shear. This requires a three-dimensional micromodel, because the primary deformations are oriented in fiber direction. In Figure 5, it is observed that the localization zone in which the strain develops in the plastic mechanism has a more simple shape as it is aligned with the boundaries of the micromodel. 


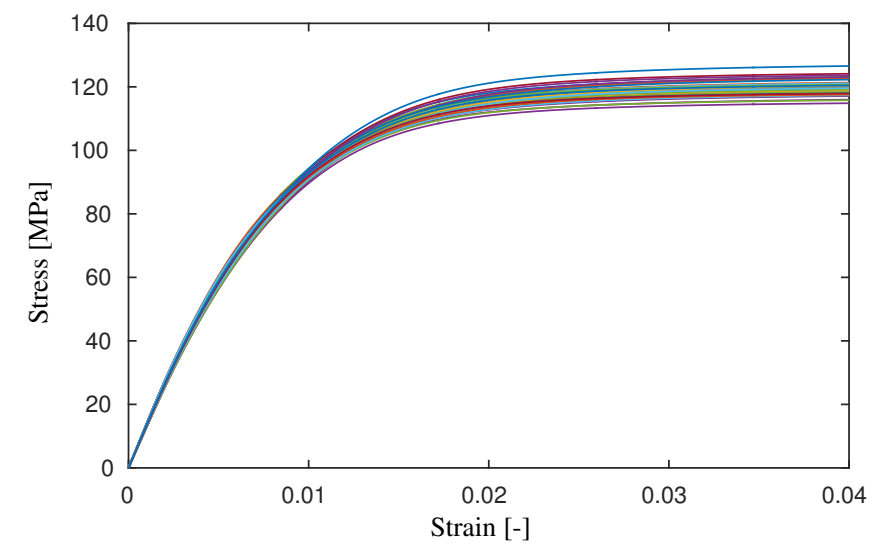

Figure 2: Stress strain response from 50 different micromodels with 25 fibers.
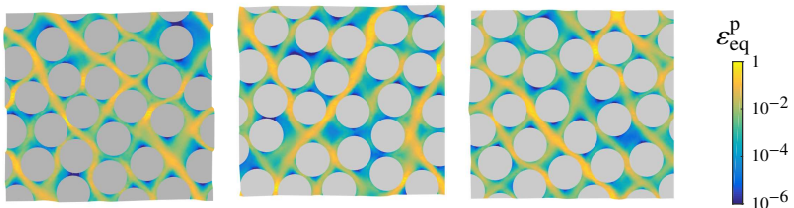

Figure 3: Final equivalent plastic strain distribution from three different micromodels with 25 fibers under tension (loaded in horizontal direction).

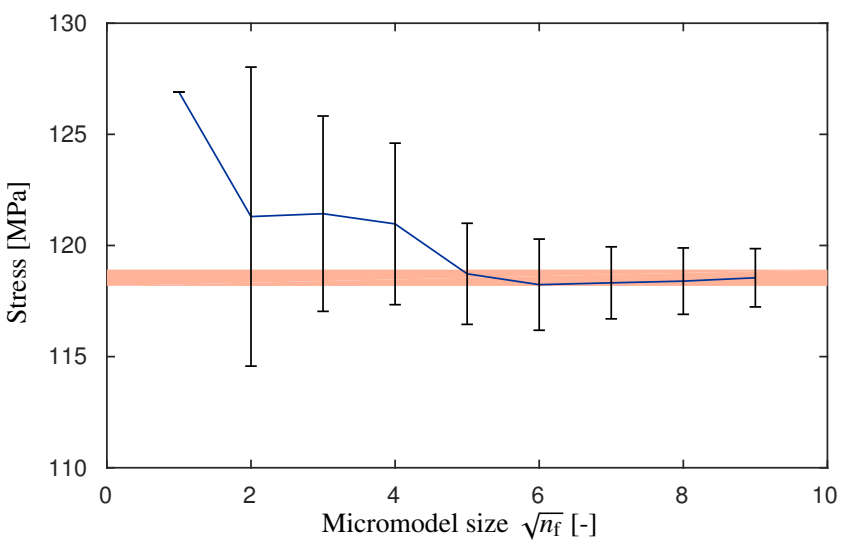

Figure 4: Predicted stress level at 0.03 strain as a function of micromodel size for uniaxial tension (plane strain); mean and standard deviation for different sizes and $95 \%$ confidence interval for $n_{\mathrm{f}}=9 \times 9$ (shading) 

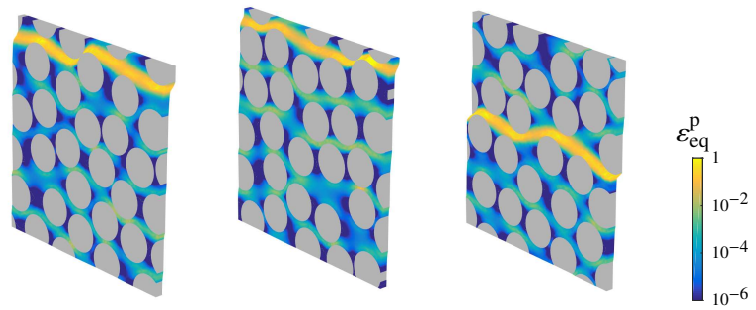

Figure 5: Final equivalent plastic strain distribution from three different micromodels under longitudinal shear with 25 fibers.

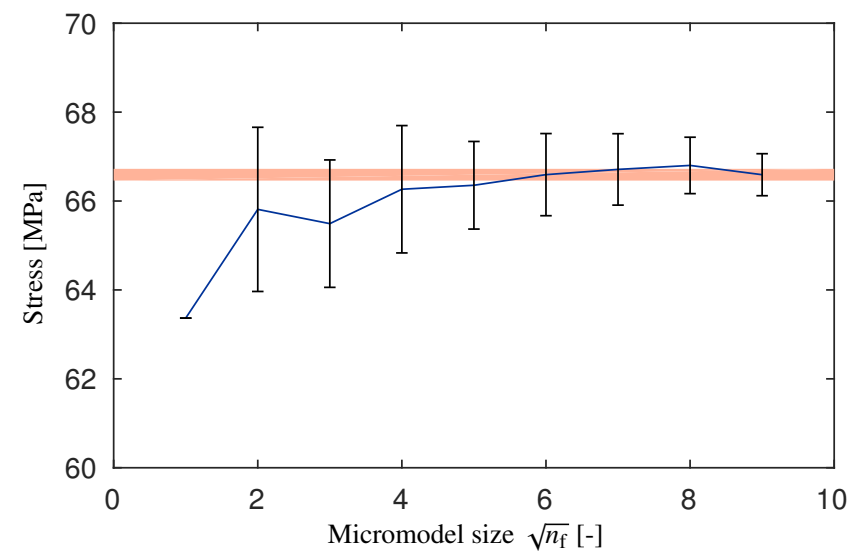

Figure 6: Predicted stress level at 0.04 strain as a function of micromodel size for longitudinal shear; mean and standard deviation for different sizes and $95 \%$ confidence interval for $n_{\mathrm{f}}=9 \times 9$ (shading)

Nevertheless, the scatter in the micromodel response remains. In Figure 6 the statistics of the final stress level (at 0.04 strain) are plotted for different micromodel sizes. A similar trend as for the tensile case is observed with decreasing but not vanishing scatter and a limited variation in mean value. The scatter in results with $9 \times 9$ fibers is smaller than for uniaxial tension. Mean final stress values from micromodels with $5 \times 5$ or $8 \times 8$ fibers are not inside the $95 \%$ interface of confidence for the mean final stress from the micromodels with $9 \times 9$ fibers, but still close.

Based on the statistical analysis, a single micromodel with $5 \times 5$ fibers is chosen as optimal in the trade-off between representativeness and efficiency for the following comparison between micromodel and mesomodel. This is a model size for which the representativeness of the results cannot be improved significantly within the investigated range of model sizes. The chosen fiber distribution had a global response close to the average from 50 specimens for for both tension and longitudinal shear.

\subsection{Calibration}

One micromodel with a given distribution of 25 fibers is used for the comparison between micromodel and mesomodel response. The three-dimensional version of the micromodel with periodic boundary conditions is used for all load cases. First, the model is subjected to six different basic load cases to generate hardening curves that are input for the mesomodel: uniaxial and biaxial tension, uniaxial and biaxial compression, and transverse and longitudinal shear.

Stress-strain curves are computed from averaged global load-displacement data. For the uniaxial simulations the axial strain in unloaded directions is also monitored. This gives information on the Poisson ratios. Transversely isotropic elasticity parameters are obtained from the first time step of the relevant load cases. The resulting values are: $E_{1}=45653 \mathrm{MPa}, E_{2}=13403 \mathrm{MPa}, G_{12}=5381 \mathrm{MPa}, v_{12}=0.231$ and $v_{23}=0.339$. 


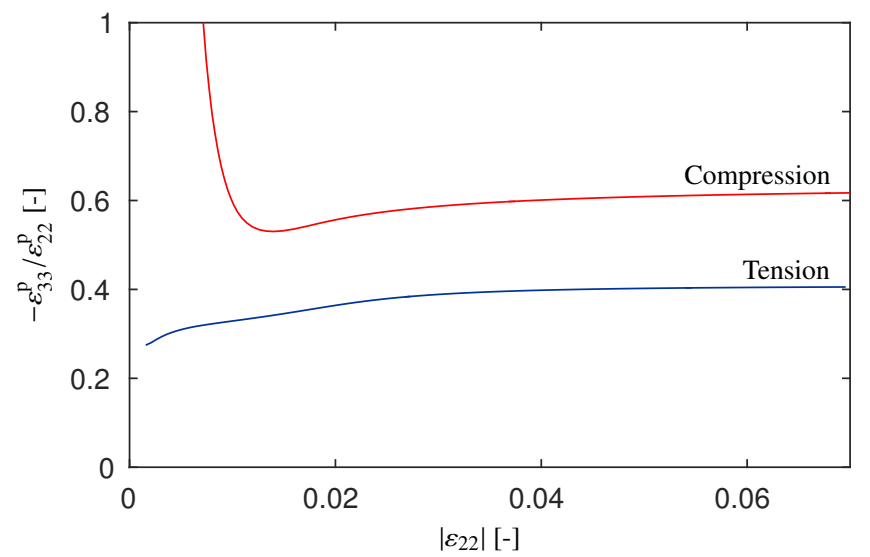

Figure 7: Plastic contraction in micromodel under uniaxial transverse tension and compression.

With the complete stress and strain vectors and the initial stiffness, it is possible to compute homogenized plastic strain vectors. From these, the plastic Poisson ratio $v_{\mathrm{p}}$ for the mesomodel is extracted. In a uniaxial test with load in $x_{2}$-direction, $v_{\mathrm{p}}$ can be computed as $v_{\mathrm{p}}=-\varepsilon_{33}^{\mathrm{p}} / \varepsilon_{22}^{\mathrm{p}}$. However, in the micromodel simulations, this ratio is not constant (see Fig. 7). Under uniaxial tension, the homogenized plastic contraction in the micromodel increases until it stabilizes at a value slightly above 0.40 . Under uniaxial compression, the plastic contraction starts out very high, then drops and eventually stabilizes around 0.62 . In the mesomodel, only one constant value can be assigned. In what follows a value of $v_{\mathrm{p}}=0.40$ will be used as input for the mesomodel. Only when contraction is specifically investigated, additional simulations will be performed with $v_{\mathrm{p}}=0.60$ as input.

With the computed homogenized plastic strain histories, the homogenized equivalent plastic strain history can be evaluated according to the definition of Vogler in Eq. (40). Alternatively, it is possible to express the plastic strain history of unloaded components by assuming a constant plastic Poisson ratio, as is done in Vogler's model. For load in 2-direction, this means:

$$
\varepsilon_{\mathrm{eq}}^{\mathrm{p}}=\varepsilon_{22}^{\mathrm{p}} \sqrt{\frac{1+v_{\mathrm{p}}^{2}}{2}}
$$

Because the assumption of constant $v_{\mathrm{p}}$ is not in agreement with the micromodel response, the two alternative procedures do not give the same result. In order to get an optimal match between mesomodel and micromodel results on calibrated cases, the relation from Eq. (68) is used in the calibration procedure. The relation between thus computed equivalent plastic strain history and stress history from the basic load cases is input for the mesomodel through the $\alpha_{i}$ variables in the yield function.

For biaxial compression, the micromodel simulation was aborted before perfect plasticity was reached due to nonconvergence of the global Newton's method.The biaxial compression input curve was based on an extrapolation of the incomplete micromechanical curve.

\subsection{Biaxial transverse stress states}

For comparison, a single element mesomodel with the calibrated Vogler plasticity model is subjected to the same load cases. Stress-strain curves from the micro and mesomodels are shown in Figures 8 and 9. In the calibrated cases, the agreement is perfect, except for the transverse shear case, which is due to the fact that the transverse shear 


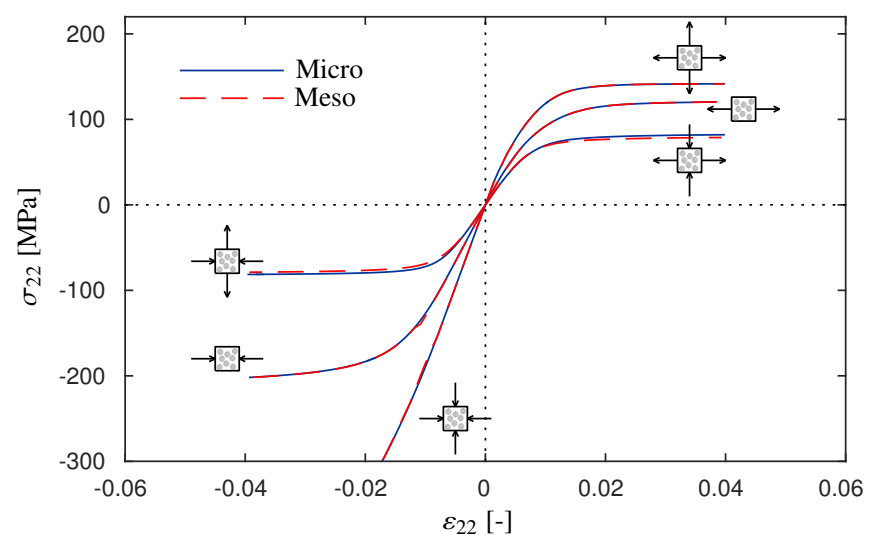

Figure 8: Stress strain curves from calibrated transverse biaxial stress states.

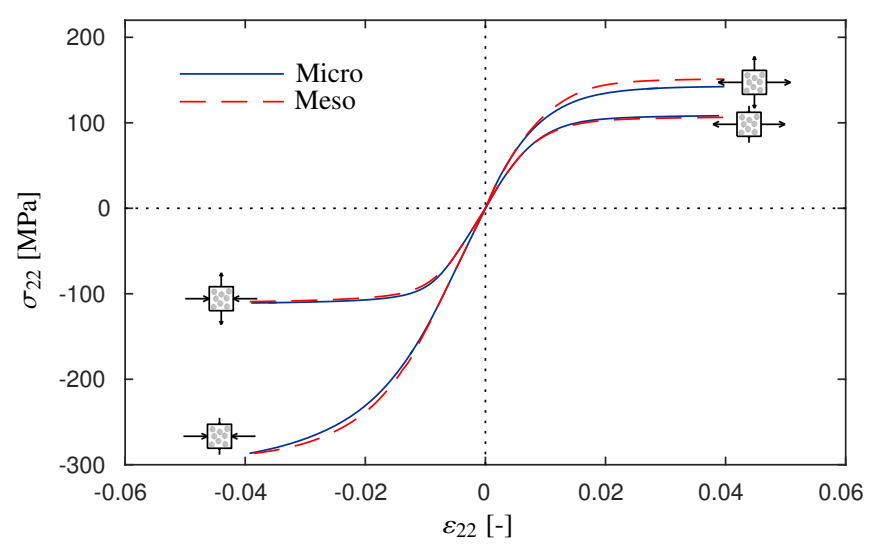

Figure 9: Stress strain curves from uncalibrated transverse biaxial stress states.

The micromodel is subjected to a series of biaxial transverse load cases. A constant averaged stress ratio $\left(\sigma_{22} / \sigma_{33}\right)$ is applied with zero averaged stress in fiber direction $\sigma_{11}$. Stresses and strains are monitored in both principal transverse directions. For comparison, a single element mesomodel with the calibrated Vogler plasticity model is subjected to the same load cases. Stress-strain curves from the micro and mesomodels are shown in Figures 8 and 9 . In the calibrated cases, the agreement is perfect, except for the transverse shear case, which is due to the fact that the transverse shear input is related to a different orientation of the stress. The small difference here points at less than perfect transverse isotropy of the micromodel. For the uncalibrated cases, the difference is larger, especially for the case that is close to biaxial tension where the final stress level in the mesomodel is $6 \%$ higher than that in the micromodel. Also for the case close to biaxial compression, a difference is visible for intermediate strain levels.

It is possible to compare the final stress levels from both models and generate the final yield envelope. For each biaxial simulation the stress level at final strain of $\sqrt{\varepsilon_{22}^{2}+\varepsilon_{33}^{2}}=0.04$ is recorded for both micro and mesomodel and the resulting envelope is visualized in Fig. 10. Symmetry along the ( $\left.\sigma_{22}=\sigma_{33}\right)$-line is indicative for transverse isotropy. In the compression-compression regime some points are missing for the micromechanical model, which is due to non-convergence of the simulations before a strain level of 0.04 was reached.

The uniqueness of the plastic Poisson ratio $v_{\mathrm{p}}$ was already discussed in the section on calibration. In order to get more insight in the actual contraction behavior predicted by both models, the strain history in the micromodel is 


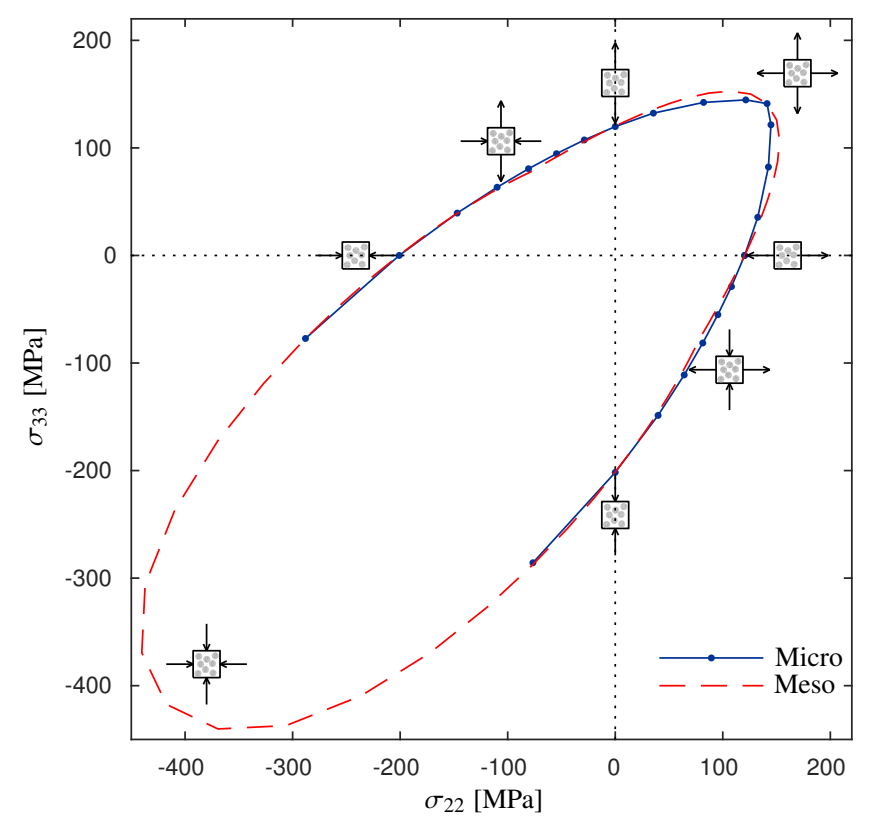

Figure 10: Envelope of final stress for different biaxial stress states.

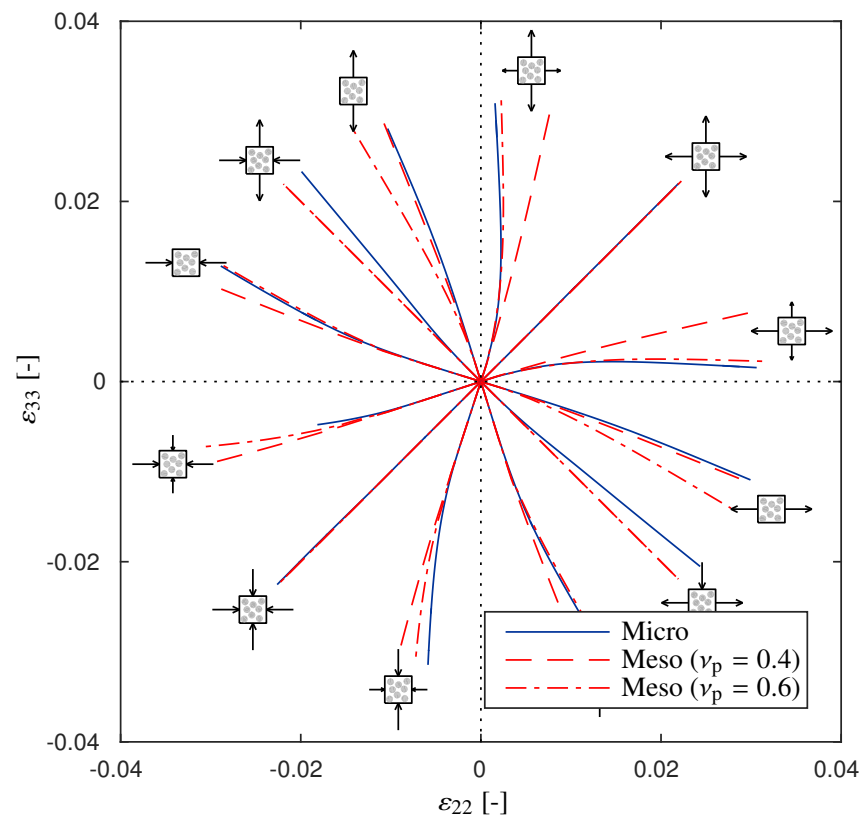

Figure 11: Strain path for different biaxial loading cases: comparison between micromodel and mesomodel with two different values for $v_{\mathrm{p}}$. 

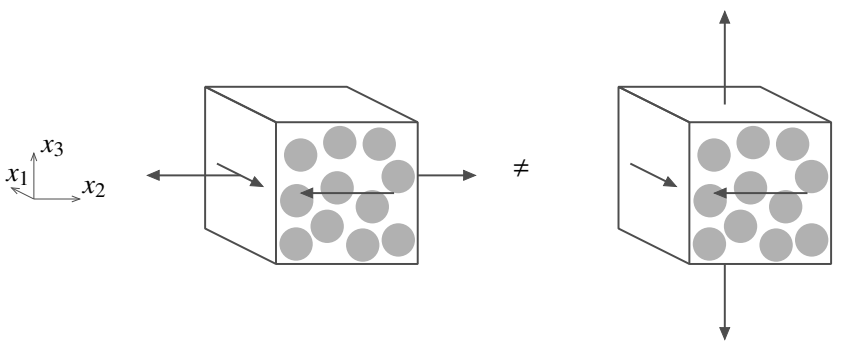

Figure 12: Different orientations of transverse uniaxial stress with respect to longitudinal shear stress.

compared to the homogenized model response for the complete range of biaxial load cases in Fig. 11. Mesomodel results are shown for two different values of $v_{\mathrm{p}}$. It is observed that for uniaxial transverse compression the value of $v_{\mathrm{p}}=0.6$ gives a better match while for the case of transverse tension, $v_{\mathrm{p}}=0.4$ works better, which is in agreement with earlier observations in Fig. 7. However, for biaxial tension with $\sigma_{22} / \sigma_{33}=\sqrt{3}$ a better match is obtained with the uniaxial compression value of $v_{\mathrm{p}}$, which means that a simple switch between compression and tension dominated behavior in the flow rule is not sufficient to repair the discrepancy. Interestingly, for pure shear, the plastic Poisson ratio does not affect the strain path in the mesomodel in which zero pressure always gives zero volumetric strain, while the micromodel does show volume increase. This volumetric strain under shear cannot be achieved with the flow rule in Eq. (38) unless the B-matrix is allowed to become asymmetric as a function of stress. It is concluded that the assumption of a single plastic Poisson ratio does not do justice to the complexity of the micromodel response. At the same time it is not easy to improve the mesomodel on this point in a reliable manner without excessively increasing the complexity of collection of required input.

\subsection{Longitudinal shear and axial transverse stress}

The most relevant scenario for plasticity in composite materials is longitudinal shear. The pure longitudinal shear behavior is an input curve. Its interaction with other plasticity inducing stress components is postulated in the way the invariant-based yield criterion is formulated in Vogler's model. A consequence of the invariant formulation is that the directionality of transverse stress and of longitudinal shear are not accounted for in their interaction. In Vogler's model it does not make a difference whether longitudinal stress component $\sigma_{12}$ is combined with transverse stress $\sigma_{22}$ or with transverse stress $\sigma_{33}$ (see Fig. 12), while for the actual composite material these two scenarios do give rise to different stress states.

In Figure 13, the envelope of final stress values under combined longitudinal shear and uniaxial transverse stress

with constant stress ratio is shown. The stress values correspond to a strain level of $\sqrt{\varepsilon_{12}^{2}+\varepsilon_{22}^{2}}=0.04$, for which the behavior is very close to perfect plasticity. It is observed that the overall agreement is fair. Especially in the tensile domain when the micromodel is loaded with $\sigma_{22}$ and $\sigma_{12}$ agreement is very good. In the compressive part, the $\left(\sigma_{22}, \sigma_{12}\right)$-micromodel overpredicts the final stress. The difference between the $\left(\sigma_{22}, \sigma_{12}\right)$-micromodel and the $\left(\sigma_{33}, \sigma_{12}\right)$-micromodel is significant, while the possibility of such difference is ignored in the mesomodel. It should be noted that the envelope visualization is a very forgiving visualization. Although the lines are visibly close, differences of up to $8 \%$ between $\left(\sigma_{22}, \sigma_{12}\right)$-micromodel and mesomodel and up to $14 \%$ between $\left(\sigma_{33}, \sigma_{12}\right)$-micromodel and mesomodel are significant. These relative differences are larger than those found for biaxial transverse stress states (Fig. 10).

The direction of plastic strain for combined longitudinal shear and transverse loading is characterized in the mesomodel when $\beta_{2}$ is assumed equal to $\beta_{1}$ in Eq. (57). In order to validate this assumption, the averaged strain 


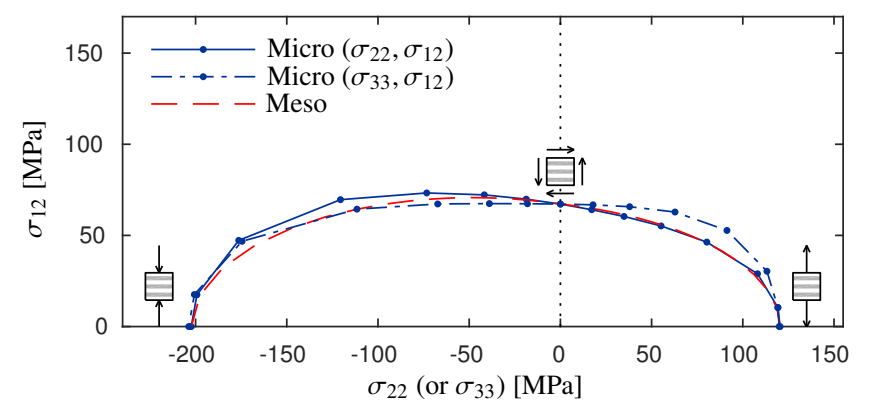

Figure 13: Final stress envelope for combined longitudinal shear and transverse axial loading.

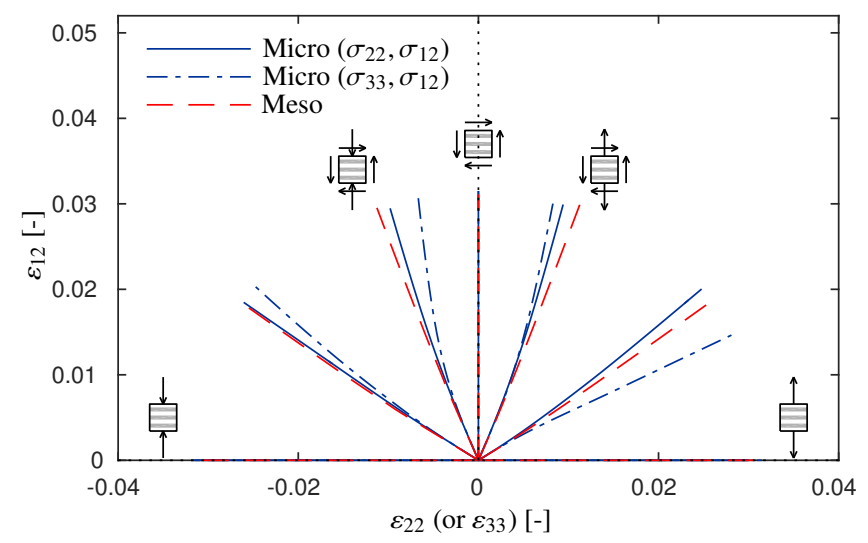

Figure 14: Strain path for combined longitudinal shear and transverse axial loading, showing results for $\sigma_{22} / \sigma_{12} \in[0, \pm 1, \pm 3.7, \pm \infty]$.

history in the micromodel responses is compared to the strain history from the mesomodel for combined longitudinal shear and transverse axial loading. In Figure 14, the strain path for a select number of stress ratios is visualized. The strain history in the pure shear and pure transverse cases does not show coupling in any of the models. The mixed cases, however, show a more challenging picture. First of all, $\left(\sigma_{22}, \sigma_{12}\right)$-micromodel corresponds quite well to the mesomodel response. In general, shear strain grows faster in the micromodels than in the mesomodel. The picture could be improved here by choosing a higher value for $\beta_{2}$ in the mesomodel. Also in the strain histories, there is a significant difference between the response of the $\left(\sigma_{22}, \sigma_{12}\right)$-micromodel and that of the $\left(\sigma_{33}, \sigma_{12}\right)$-micromodel. If one were to improve the agreement between the mesomodel and the $\left(\sigma_{22}, \sigma_{12}\right)$-micromodel by changing the value of $\beta_{2}$ in the mesomodel, this would for some stress combinations make the agreement between $\left(\sigma_{33}, \sigma_{12}\right)$-micromodel less favorable.

\subsection{Influence of stress in fiber direction}

The stress in fiber direction is intentionally kept out of consideration in Vogler's plasticity model for the composite material. Nevertheless, the actual plasticity taking place in the matrix material is a three-dimensional process that is influenced by loading in all directions. To investigate the influence of stress in fiber direction on plasticity in transverse and longitudinal directions, the meso and micromodels are subjected to stress histories that include stress in fiber direction. Constant stress ratio simulations are performed which combine stress in fiber direction, $\sigma_{11}$, with stress in a matrix dominated direction. Firstly, $\sigma_{11}$ is combined with longitudinal shear $\sigma_{12}$ and secondly with transverse uniaxial stress $\sigma_{22}$. 


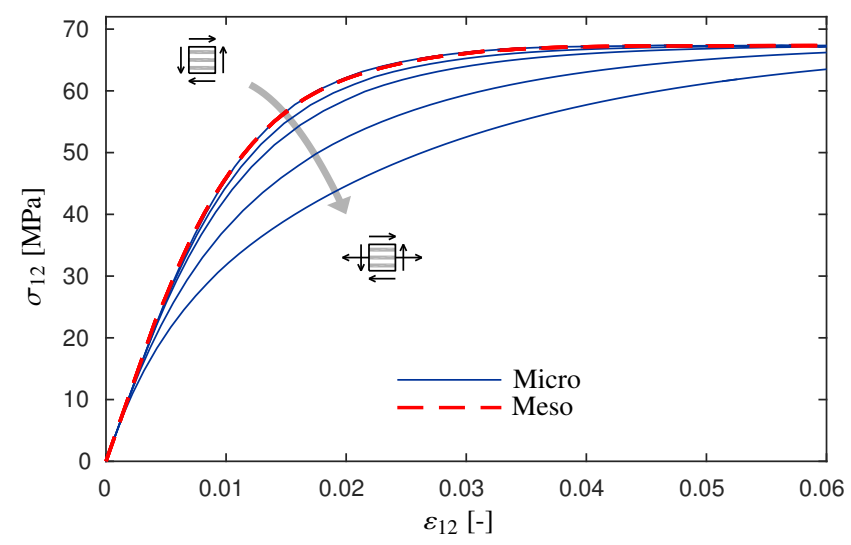

Figure 15: Shear stress-strain curve for combined longitudinal shear and longitudinal axial tension $\left(\sigma_{11} / \sigma_{12} \in[57,29,11,6,0]\right)$.

The stress-strain curve in fiber direction remains nearly linear because the linear elastic fibers dominate the response. Therefore, the influence of stress in fiber direction is assessed by examining the stress-strain curves in matrix dominated directions. In Figures 15 and 16 the shear response for combined longitudinal shear and longitudinal axial loading is shown; in the first image for tension in fiber direction and in the second for compression in fiber direction. For the mesomodel only one curve is visible. In fact, all curves for the mesomodel coincide as the shear response is completely independent of the longitudinal stress. In contrast, the micromodel results show a clear influence of longitudinal stress on the shear response. The more the composite material is loaded in fiber direction, the lower the shear stress is for the same shear strain level. Plasticity becomes significant for much lower shear stress levels. Eventually, the same final shear stress level is reached for the perfectly plastic tail, but the rate with which this value is approached is strongly dependent on stress in fiber direction. Note that the stress ratios are high, but that values close to $3000 \mathrm{MPa}$ are reported for the tensile strength of carbon/epoxy [27] (depending on the loaded volume) which means that with the highest stress ratio considered of $\sigma_{11} / \sigma_{12}=57$, a shear stress of about $50 \mathrm{MPa}$ can still be attained prior to fiber failure.

In the matrix material in the micromodel, the direction of plastic strain is a combination of $\varepsilon_{12}^{\mathrm{p}}$ and $\varepsilon_{11}^{\mathrm{p}}$. However, the effect of these two plastic strain components on the stress distribution is different, because in 11-direction fibers and matrix are loaded in parallel, while in 12-direction they are loaded approximately in series. The plastic strain in the matrix in 11-direction causes a redistribution of stress from the matrix to the fibers. While the $\sigma_{11}$ component in the matrix reduces, the influence of the applied total $\sigma_{11}$ on the matrix plasticity disappears. This explains the observation that the final shear stress level is independent of the applied stress ratio. In the localization band that forms the plastic mechanism, the stress state eventually becomes such that plastic strain only keeps growing in 12 direction, irrespective of the globally applied stress ratio.

For compression in fiber direction (Fig. 16) the influence of $\sigma_{11}$ on the longitudinal shear response is less pronounced. In fact, for moderate longitudinal compression, the shear stress increases slightly, similarly to what happens for moderate transverse compression (see Fig. 13). Only for extreme stress ratios a strong effect is observed. Here it should be noted that the compression strength in fiber direction of the considered composite is generally lower than the tensile strength, and that this compression strength is further reduced in presence of longitudinal shear. As a consequence, the influence of compression in fiber direction on longitudinal shear remains limited for realistic stress values.

For combined longitudinal stress and transverse uniaxial stress, transverse stress-strain curves are shown in Fig- 


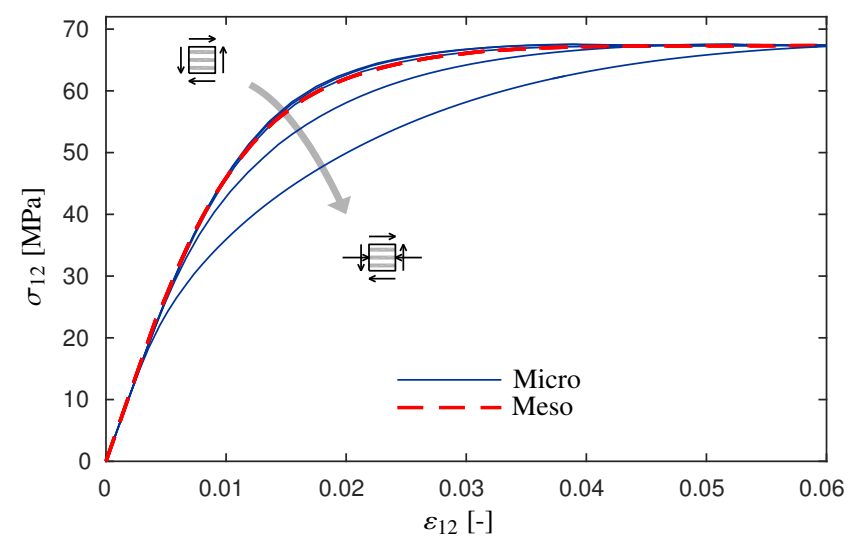

Figure 16: Shear stress-strain curve for combined longitudinal shear and longitudinal axial compression $\left(\sigma_{11} / \sigma_{12} \in[-57,-29,-11,-6,0]\right)$.

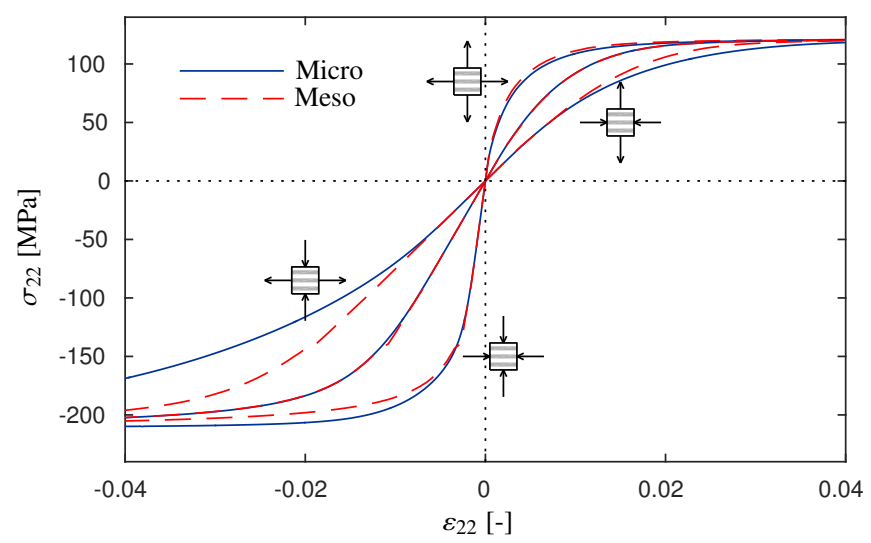

Figure 17: Transverse stress-strain curves for transverse/longitudinal biaxial stress states $\left(\sigma_{11} / \sigma_{22} \in[-11,0,11]\right)$.

ure 17. Here, the mesomodel curves also differ from one another, which is due to the coupling between the axial stresses and strains in elasticity. The elasticity effect is well-captured, which should not come as a surprise, because homogenization works very well when it comes to elasticity in orthotropic materials. For the plastic part of the curves, there is some difference between the mesomodel and micromodel responses, especially for the cases where the two active stress components have an opposite sign. The case with transverse compression and longitudinal tension has the most significant difference, which is troublesome because under transverse compression plasticity is more likely to become significant prior to failure than it is under transverse tension.

\section{Conclusions}

A detailed comparison between a micromodel based on Melro's plasticity model and a homogenized mesomodel based on Vogler's plasticity model has been performed. First, the size of micromodel needed for representativeness of the response of a micromodel with elastic-plastic matrix was investigated. It was found that the mean response stressstrain curve converges with an RVE size of $5 \times 5$ fibers. However, the statistical scatter did not vanish for RVE sizes with up to $9 \times 9$ fibers, which means that a single RVE of that size can still not be taken as completely representative.

The mesomodel was calibrated based on basic stress-strain curves obtained from a single micromodel with $5 \times 5$ fibers. The same micromodel was subsequently subjected to a range of different stress states and differences with 
the homogenized model response were investigated. Stress strain curves for biaxial transverse loading agree well. However, when inspecting the plastic contraction for different biaxial stress states it was found that using a single plastic Poisson ratio in the mesomodel does not allow for an accurate reproduction of the micromechanically observed plastic contraction under general load conditions.

Concerning combined longitudinal shear and transverse loading, it was observed that a significant difference in micromodel response is obtained depending on whether the transverse and longitudinal shear loading are in the same plane or not. This distinction is lost in the invariant-based formulation of the mesomodel.

Influence of the stress in fiber direction on plasticity was deliberately left out of the mesolevel plasticity model. However, the micromodel with three-dimensional pressure dependent matrix plasticity law did predict influence of stress in fiber direction on the transverse or longitudinal shear behavior. This influence was most strong for the combination of longitudinal shear with tension in fiber direction. Longitudinal shear is the scenario for which plasticity is most relevant in laminates, while laminates are optimally designed when they carry load in fiber direction. Therefore, lack of accounting for this interaction is considered the most serious shortcoming of the mesomodel.

\section{Acknowledgment}

Financial support by the Netherlands Technology Foundation STW (under grant 12502) is gratefully acknowledged.

\section{References}

\section{References}

[1] S. W. Tsai and E. M. Wu. A general theory of strength for anisotropic materials. J Compos Mater, 5(1):58-80, 1971.

[2] Z. Hashin. Failure criteria for unidirectional fiber composites. J Appl Mech, 47(2):329-334, 1980.

[3] A. Puck and H. Schürmann. Failure analysis of FRP laminates by means of physically based phenomenological models. Compos Sci Technol, 58(7):1045-1067, 1998.

[4] C. G. Dávila, P. P. Camanho, and C. A. Rose. Failure criteria for FRP laminates. J Compos Mater, 39(4):323-345, 2005.

[5] P. P. Camanho, A. Arteiro, A. R. Melro, G. Catalanotti, and M. Vogler. Three-dimensional invariant-based failure criteria for fibre-reinforced composites. Int J Solids Struct, 55:92-107, 2015.

[6] E. Totry, C. González, and J. LLorca. Failure locus of fiber-reinforced composites under transverse compression and out-of-plane shear. Compos Sci Technol, 68(3-4):829-839, 2008.

[7] E. Totry, C. González, and J. LLorca. Prediction of the failure locus of C/PEEK composites under transverse compression and longitudinal shear through computational micromechanics. Compos Sci Technol, 68(15-16):3128-3136, 2008.

[8] T. J. Vaughan and C. T. McCarthy. A micromechanical study on the effect of intra-ply properties on transverse shear fracture in fibre reinforced composites. Compos Part A, 42:1217-1228, 2011.

[9] A. R. Melro, P. P. Camanho, F. M. Andrade Pires, and S. T. Pinho. Micromechanical analysis of polymer composites reinforced by unidirectional fibres: Part II - Micromechanical analyses. Int J Solids Struct, 50:1906-1915, 2013.

[10] M. R. Wisnom and F.-K. Chang. Modelling of splitting and delamination in notched cross-ply laminates. Compos Sci Technol, 60(15):28492856, 2000.

[11] F. P. van der Meer, C. Oliver, and L. J. Sluys. Computational analysis of progressive failure in a notched laminate including shear nonlinearity and fiber failure. Compos Sci Technol, 70(4):692-700, 2010.

[12] P. P. Camanho, C. G. Dávila, S. T. Pinho, L. Iannucci, and P. Robinson. Prediction of in situ strengths and matrix cracking in composites under transverse tension and in-plane shear. Compos Part A, 37(2):165-176, 2006.

[13] W. Van Paepegem, I. De Baere, and J. Degrieck. Modelling the nonlinear shear stress-strain response of glass fibre-reinforced composites. Part I: Experimental results. Compos Sci Technol, 66(10):1455-1464, 2006.

[14] E. Ghorbel. A viscoplastic constitutive model for polymeric materials. Int J Plast, 24:2032-2058, 2008.

[15] R. Hill. The Mathematical Theory of Plasticity. Oxford University Press, London, 1950.

[16] X. Li, P. G. Duxbury, and P. Lyons. Considerations for the application and numerical implementation of strain hardening with the Hoffman yield criterion. Comput Struct, 52(4):633-644, 1994 
[17] F. P. van der Meer and L. J. Sluys. Continuum models for the analysis of progressive failure in composite laminates. J Compos Mater, 43(20):2131-2156, 2009.

[18] M. Vogler, R. Rolfes, and P. P. Camanho. Modeling the inelastic deformation and fracture of polymer composites - Part I: Plasticity model. Mech Mater, 59:50-64, 2013.

[19] F. Feyel and J. L. Chaboche. $\mathrm{FE}^{2}$ multiscale approach for modelling the elastoviscoplastic behaviour of long fibre SiC/Ti composite materials. Comput Method Appl Mech Eng, 183:309-330, 2000.

[20] A. R. Melro, P. P. Camanho, F. M. Andrade Pires, and S. T. Pinho. Micromechanical analysis of polymer composites reinforced by unidirectional fibres: Part I - Constitutive modelling. Int J Solids Struct, 50:1897-1905, 2013.

[21] A. R. Melro. Analytical and Numerical Modelling of Damage and Fracture in Advanced Composites. PhD thesis, FEUP, 2011.

[22] F. P. van der Meer and L. J. Sluys. Interaction Between Intraply and Interply Failure in Laminates. In P. P. Camanho, S. T. Pinho, C. G. Dávila, and J. J. C. Remmers, editors, Mechanical Response of Composites, Computational Methods in Applied Sciences, chapter 7, pages 141-160. Springer, 2008.

[23] A. R. Melro, P. P. Camanho, and S. T. Pinho. Influence of geometrical parameters on the elastic response of unidirectional composite materials. Compos Struct, 94:3223-3231, 2012.

[24] C. González and J. LLorca. Mechanical behavior of unidirectional fiber-reinforced polymers under transverse compression: Microscopic mechanisms and modelling. Compos Sci Technol, 67(13):2795-2806, 2007.

[25] L. P. Canal, J. Segurado, and J. LLorca. Failure surface of epoxy-modified fiber-reinforced composites under transverse tension and out-ofplane shear. Int J Solids Struct, 46(11-12):2265-2274, 2009.

[26] C. Geuzaine and J.-F. Remacle. Gmsh: A Three-dimensional Finite Element Mesh Generator with Built-in Pre- and Post-processing Facilities. Int J Numer Method Eng, 79(11):1309-1331, 2009.

[27] M. R. Wisnom, B. Khan, and S. R. Hallett. Size effects in unnotched tensile strength of unidirectional and quasi-isotropic carbon/epoxy composites. Compos Struct, 84(1):21-28, 2008. 